\title{
Variation in sex ratio and evolutionary rate of hemiclonal Rana esculenta populations
}

\author{
CHRISTIAN SOM ${ }^{1,2}$ and HEINZ-ULRICH REYER ${ }^{1, *}$ \\ ${ }^{1}$ Zoological Institute, University of Zurich, Winterthurerstrasse 190, CH-8057, Zurich, Switzerland; \\ ${ }^{2}$ EMPA St. Gallen, Technology \& Society Lab, Lerchenfeldstrasse 5, CH-9014, St. Gallen, \\ Switzerland (*author for correspondence, tel.: +41-44-635-49-80; fax: +41-44-635-68-21; \\ e-mail: ulireyer@zool.unizh.ch)
}

Received 18 November 2004; Accepted 29 November 2005

Co-ordinating editor: L. Hurst

\begin{abstract}
In many plant and animal taxa mutation rates are higher in males than in females. As a result, the evolutionary speed of genes depends on how much time they spend in either sex. Usually, this time differs between genes located on sex chromosomes but not between those on autosomes. Here we present an unusual system with a partially sex-linked inheritance of autosomes: the hemiclonal frog Rana esculenta (E) which is originally a hybrid between the sexual species $R$. lessonae $(\mathrm{L})$ and $R$. ridibunda $(\mathrm{R})$. Rana esculenta excludes the $\mathrm{L}$ genome prior to meiosis, produces eggs or sperm containing an unrecombined $\mathrm{R}$ genome and restores hybridity by mating with $R$. lessonae ('hybridogenesis'). Matings between $\mathrm{L}$ males and $\mathrm{E}$ females result in offspring with an even sex ratio, whereas the reverse combination produces only daughters. The extent of the resulting female bias and the proportion that $\mathrm{R}$ alleles have spent in either sex depend on the relative survival $(b)$ and the relative reproductive contribution $(a)$ of $\mathrm{E}$ males vs. E females. In this paper, we analyze mathematically how different combinations of $a$ and $b$ influence the sex ratio in $R$. esculenta populations and, combined with the male/female mutation rate ratio $(\alpha)$, the evolutionary rate of the clonally transmitted $\mathrm{R}$ genome. We find that this rate is higher than in an asexual population and lower than in a sexual one. Hence, clonal diversity through new mutations is more easily achievable than in purely asexual species. In contrast, the occurrence and accumulation of deleterious mutations is lower than in a comparable sexual species. We conclude that these intermediate mutation rates improve the ecological and evolutionary potential of hemiclonal organisms, and we draw attention to the implications for the use of microsatellites.
\end{abstract}

Key words: evolutionary rate, hybridogenesis, Rana esculenta, sex biased mutation rate, sex ratio

\section{Introduction}

In the first half of the 20th century, Haldane $(1935,1947)$ demonstrated that most mutations generating haemophilia in humans occurred in the male germ line. Meanwhile, sex biases in mutation rates have been found for several loci in fishes, birds, rodents and primates, including humans. In these groups, the ratio of male to female mutation rate $(\alpha)$ ranges from 1 to 10 , with differences between sexes being highest in species which produce many male gametes, such 
as wind pollinated plants or externally fertilizing animals (Miyata et al., 1987; Redfield, 1994; Hurst and Ellegren, 1998; McVean, 2000; Makova and Li, 2002; Ellegren and Fridolfsson, 2003). Since spermatogonia divide throughout the whole life of males whereas oogenesis in females is largely complete at birth, the observed sex difference has been interpreted as evidence that new mutations occur during DNA replication. This led to the suggestion that evolution is 'male driven'.

Miyata et al. (1987) suggested testing for a difference in male and female mutation rates by comparing the evolutionary rates of sex chromosomes and autosomes (see also Kirkpatrick and Hall, 2004). If male and female mutation rates are different, evolutionary rates of neutral mutations should be highest for $\mathrm{Y}$-chromosomes which only occur in males and lowest for $\mathrm{X}$ chromosomes which spend about $2 / 3$ of their history in females. The evolutionary rate of neutral mutations on autosomes should approximately equal the average of male and female mutation rates, because autosomes spend about equal times in both sexes. Here, we extend Miyata's et al. (1987) approach for studying sexspecific evolutionary rates to an unusual species, the edible frog (Rana esculenta), in which autosomes, too, spend more time in females than in males. Because of this sex-linked inheritance, mutations rates are expected to be lower than the average between high male and low female rates that is predicted for 'normal' species.

Rana esculenta originated from hybridization between the pool frog Rana lessonae (L) and the lake frog $R$. ridibunda (R). During gametogenesis, $R$. esculenta excludes one parental genome (usually $\mathrm{L}$ ) from the germ line prior to meiosis (Berger, 1977; Graf and Müller, 1979; Uzzell et al., 1980) and transmits only an unrecombined, i.e. clonal $\mathrm{R}$ genome to eggs or sperm ('hybridogenesis'; Schultz, 1969). Hence, $\mathrm{E} \times \mathrm{E}$ matings result in $R$. ridibunda tadpoles, but these do not survive (Berger, 1976; Graf and Müller, 1979; Semlitsch and Reyer, 1992), unless the parents possess and transmit different clonal R genomes (Vorburger, 2001; Guex et al., 2002). This suggests that the usual inviability of offspring from $\mathrm{E} \times \mathrm{E}$ matings is due to homozygosity for deleterious alleles that have accumulated on the clonally transmitted $\mathrm{R}$ genome through 'Muller's ratchet' (Muller, 1964; Felsenstein, 1974; Charlesworth and Charlesworth, 1997).

In areas where $R$. ridibunda is absent, the only way for $R$. esculenta to propagate itself is to backcross with $R$. lessonae to regain the premeiotically eliminated $\mathrm{L}$ genome. While $R$. esculenta females mating with $R$. lessonae males $(\mathrm{E} \times \mathrm{L})$ produce offspring with an even sex ratio, male $R$. esculenta mating with female $R$. lessonae $(\mathrm{L} \times \mathrm{E})$ sire only daughters. This sex bias is a direct result of the combined effects of sex determination, hybridogenesis and mating behaviour (Fig. 1). Sex is determined by an autosomal $\mathrm{XX}-\mathrm{XY}$ system with males being heterogametic. Since - for size and behavioural reasons - primary 


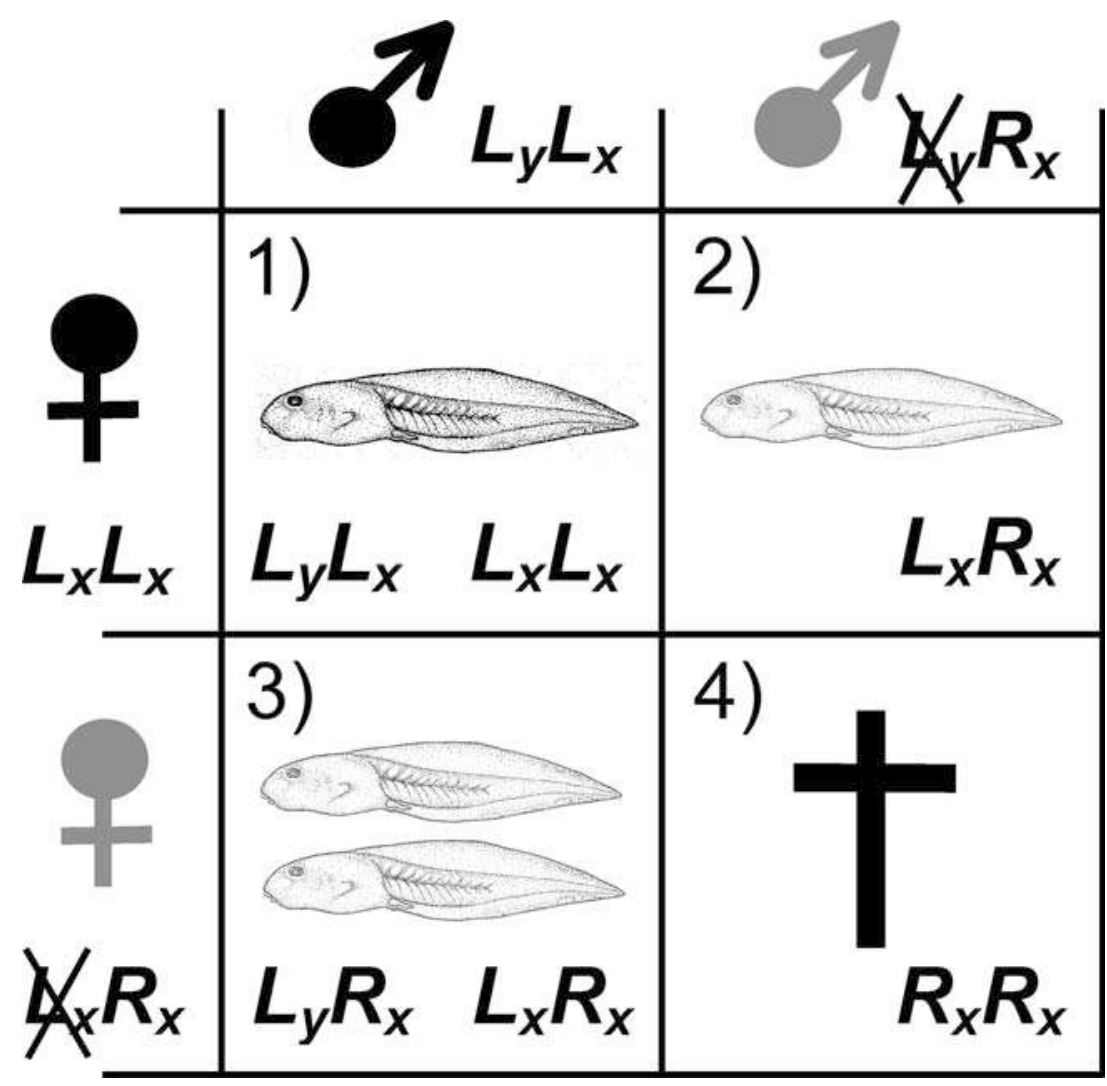

Figure 1. Possible mating combinations and resulting offspring (cells 1-4) in mixed populations of $R$. lessonae (genotype LL) and $R$. esculenta (genotype LR). The crossed out L (W) indicates that the hybrid $R$. esculenta eliminates the parental L-genome premeiotically. Hence, it produces eggs and sperm containing the R-genome only. Since the hybrid's ridibunda-genome is of maternal $\left(\mathrm{R}_{\mathrm{x}}\right)$ and its lessonae-genome of paternal origin $\left(\mathrm{L}_{\mathrm{y}}\right)$, premeiotic elimination of the latter results in the exclusive production of X-gametes. Consequently, matings with $\mathrm{E}$ males (second column) lead to daughters only $\left(L_{x} R_{x}, R_{x} R_{x}\right)$ whereas those with $L$ males (first column) produce equal numbers of male $\left(L_{y} L_{x}, L_{y} R_{x}\right)$ and female offspring $\left(L_{x} L_{x}, L_{x} R_{x}\right)$. The higher number of tadpoles in cell 3 than in cells 1 and 2 illustrates the higher fecundity of LR- compared to LL-females; the † in cell 4 indicates that the resulting $R$. ridibunda (RR) tadpoles do not survive to metamorphosis.

hybridizations arise mainly from matings between $\mathrm{L}$ males and $\mathrm{R}$ females, rather than L females and $\mathrm{R}$ males (Tunner, 1974; Berger et al., 1988), premeiotic exclusion of the $\mathrm{L}$ genome removes the male determining factor from the germ line. As a result, E male sperm only contains the maternally inherited X-ridibunda chromosomes.

If the $\mathrm{E} \times \mathrm{L}$ and $\mathrm{L} \times \mathrm{E}$ matings were equally successful in producing offspring, a hybrid female would produce a daughter and a son and a hybrid male would produce two daughters. However, this does not produce a sex ratio of $1 \mathrm{~m}: 3 \mathrm{f}$ in 
the population. The three daughters and the one son from the second generation would produce five granddaughters (three from the daughters + two from the son) and three grandsons, yielding a sex ratio of 3:5 in the third generation. This continues until eventually a stable sex ratio of $1 \mathrm{~m}: 2 \mathrm{f}$ is reached - provided that survival does not differ between the sexes. If all sons but only one of the three daughters survive, the population sex ratio at reproduction would always be 1:1 instead of the $1 \mathrm{~m}: 2 \mathrm{f}$. A ratio of $1: 1$ would also result if all $R$. esculenta males were infertile or otherwise not successful. In that situation, hybrid offspring would exclusively result from $E$ females mating with $L$ males $(E \times L)$ which produce an equal sex ratio.

Under both scenarios (i.e. no E male success or equal $\mathrm{E}$ male and $\mathrm{E}$ female success), the evolutionary rate of autosomes is by no means the average between the male and female mutation rates. With no male success, an autosome present in the 10th generation of the E population MUST have passed its former 9 'lifes' in an E female. With equal success, R autosomes from $R$. esculenta females are passed on to both sexes (Fig. 1, cell 3) but those from $R$. esculenta males always end up in a daughter (Fig. 1, cell 2). Hence, on average, $\mathrm{R}$ autosomes have spent more time in females than in males during their history. Since, for neutral mutations (to which we restrict our analyzes in this paper), the mutation rate equals evolutionary rate, the hybrid autosomes' evolutionary rate is, therefore, no longer the average of male and female mutation rates, but lower.

These numerical examples illustrate that sex ratio and the evolutionary rate of E populations depend on how much the E male fraction contributes to the next generation. This, in turn, depends on a combination of sex specific reproductive success and sex specific survival until reproduction. Empirical studies have shown that the two parameters do, indeed, differ between the sexes. Reproductive success is higher in female than in male $R$. esculenta due to (a) female avoidance of E males but no male avoidance of $E$ females (Abt and Reyer, 1993, Reyer et al., 1999; Roesli and Reyer, 2000; Engeler and Reyer, 2001), (b) higher fecundity of E females mating with L males than of L females mating with E males (Berger and Uzzell, 1980; Reyer et al., 1999), and (c) reduced fertility in hybrid males, ranging from equal fertilization rate of eggs by E- than by L-sperm to total sterility of E males (Günther, 1990; Reyer et al., 2003). In terms of survival, sex differences in $R$. esculenta seem to be less pronounced. One study detected no differences between males and females (Anholt et al., 2003) whereas another study found a slightly better survival in males than in females (Holenweg Peter, 2001).

In this article, we investigate through a mathematical analysis how sex differences in survival and reproductive success influence the sex ratio and, thus, the evolutionary rate of $R$. esculenta populations if male and female mutation rates are different. 


\section{Methods}

An analytic solution for the effects of sex specific reproductive success and survival until reproduction on sex ratio and the evolutionary rate of E populations can be found the following way:

Assume that the E female's fertility is 2 (a son and a daughter) and the factor $a$ denotes the relative success of $\mathrm{E}$ males in producing offspring compared to $\mathrm{E}$ females. Let $b$ be the relative probability of survival until reproduction of $\mathrm{E}$ males compared to $\mathrm{E}$ females. Then, with generations not overlapping, the reproductive males in generation $t+1$ can be calculated as

$$
m(t+1)=b * f(t) * 1
$$

i.e. each female $f(t)$ from generation $t$ gives birth to one son which survives with the probability of $b$. The number of females in generation $t+1$ can be calculated as

$$
f(t+1)=f(t) * 1+m(t) * 2 * a
$$

i.e. one daughter from each female plus two daughters from males $m(t)$.

Introducing $s$ as the sex ratio $m / f$ at the time of reproduction yields

$$
s(t+1)=\frac{b f(t)}{f(t)+2 a m(t)}
$$

Since $m(t)=s(t)^{*} f(t)$, the sex ratio in generation $t+1$ will be

$$
s(t+1)=\frac{b f(t)}{f(t)+2 * a * s(t) * f(t)}=\frac{b}{1+2 a s(t)}
$$

At equilibrium, the sex ratio remains stable from generation to generation. Therefore,

$$
s(t+1)=s(t) \text { and } s=\frac{b}{1+2 a s} .
$$

Solving for $s$ gives the result

$$
s=\frac{-1 \pm \sqrt{1+8 a b}}{4 a}
$$

only the positive solution is of biological significance.

Applying Equations (1) and (2) to the evolutionary rate of autosomes in $R$. esculenta and assuming that females have a mutation rate of 1 (defined as the number of new mutations per individual per generation) and males a mutation rate of $\alpha$, newborn sons inherit a total of $f(t)^{*} 1$ new mutations and a fraction $b$ of these sons will survive until reproduction; newborn daughters that originate from $\mathrm{E}$ mothers inherit a total of $f(t)^{*} 1$ new mutations as well and 
newborn daughters that originate from $\mathrm{E}$ fathers inherit a total of $\alpha^{*} 2 a m(t)$ new mutations.

The total number of new mutations in the new generation at reproduction is thus $\alpha 2 a m(t)+(1+b)^{*} f(t)$. The per capita evolutionary rate, $u$, is the total number of transferred new mutations still present at reproduction divided by the population size at reproduction:

$$
u(t+1)=\frac{\alpha 2 a m(t)+(1+b) f(t)}{2 a m(t)+(1+b) f(t)}
$$

Under the biologically reasonable assumption that the evolving E population eventually develops a stable sex ratio and the size of the population remains constant, it follows that

$$
m(t+1)=m(t) \text { and } f(t+1)=f(t) \text { and } u(t)=u(t+1) .
$$

The evolutionary rate of a $R$. esculenta population can then be expressed as a function of $a$ and $b$ alone, namely

$$
u=\frac{2-\alpha+\alpha \sqrt{1+8 a b}+2 b}{1+\sqrt{1+8 a b}+2 b}
$$

Note that if $\alpha=1$ then $u=1$ and thus independent of $a$ and $b$ and the resulting sex ratio, as expected if male and female mutation rates were equal.

\section{Results}

Figure 2 shows the sex ratio of a $R$. esculenta population living in sympatry with $R$. lessonae as the result of the success of $\mathrm{E}$ males in siring offspring relative to the success of $\mathrm{E}$ females $(a)$ and the relative survival of male juveniles until reproduction $(b)$. With the parameter range used for this analysis (males being half as successful to being twice as successful as females for both parameters), the sex ratio ranges from 1:1 to a female bias of 1:4. The female bias in the sex ratio increases with decreasing survival of males (decreasing $b$ ). This is not as trivial as it may at first seem. Remember that a low E male frequency works towards a more even sex ratio in the next generation, as $\mathrm{E}$ females that mate with $\mathrm{L}$ males produce offspring with an even sex ratio whereas successfully reproducing E males produce all female offspring. This is the reason why the female bias also increases with increasing reproductive success of males (increasing $a$ ).

With even success in siring offspring and even survival of both sexes $(\log 2(a)$ and $\log 2(b)=0)$, females are still twice as abundant as males. Even if males are twice as successful in siring offspring and male survival is twice as high as female survival, the resulting population sex ratio is still female biased 


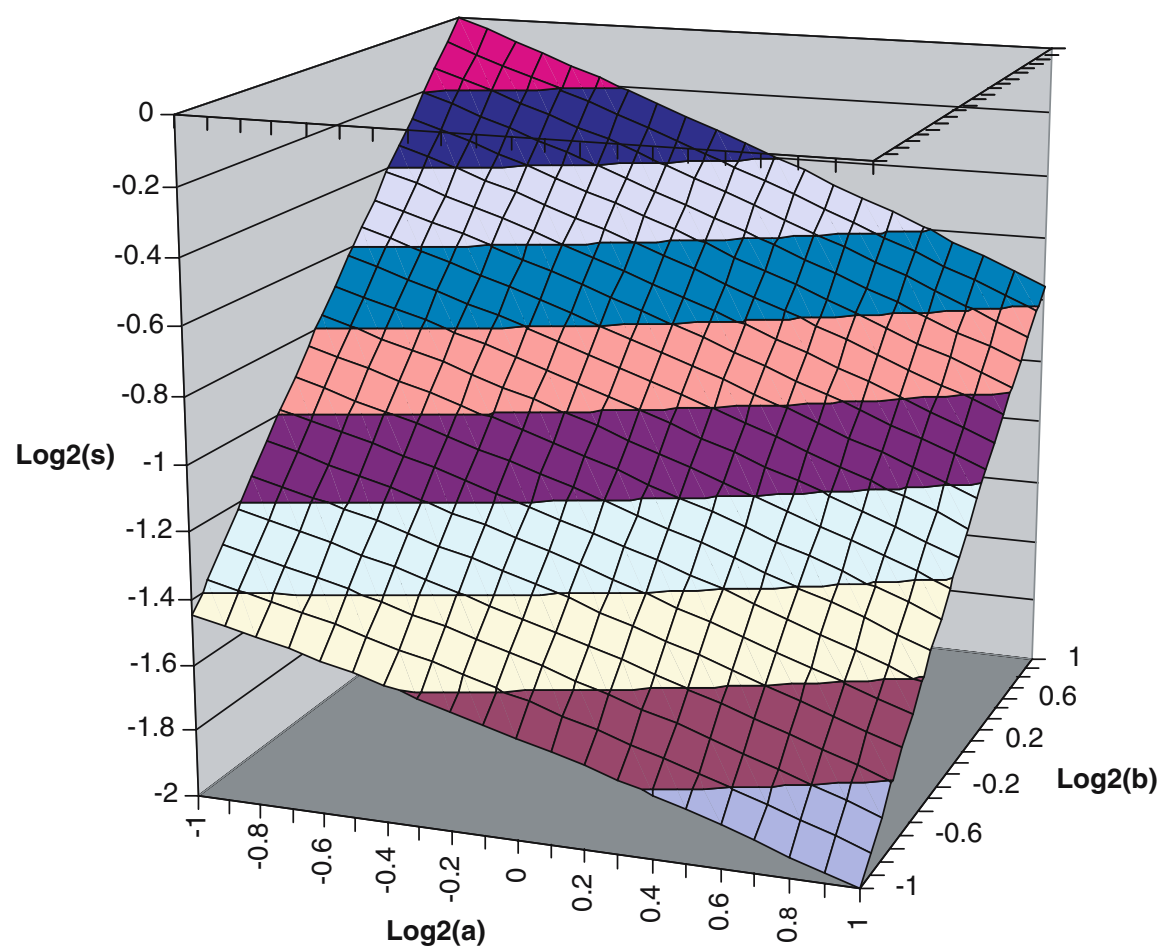

Figure 2. Dependency of the sex ratio $(s)$ on the relative reproductive success $(a)$ and the relative survival rate until reproduction $(b)$ of $R$. esculenta males compared to $R$. esculenta females. Parameters $a$ and $b$ are varied from males being half as successful as females to those being twice as successful as females. Since all parameter are defined as ratios, all axes are plotted in a $\log 2$ scale.

$(\log 2(a)$ and $\log 2(b)=1)$. It is noteworthy that different combinations of $a$ and $b$ can produce the same sex ratio: if $c$ is the constant sex ratio then all $a-b$ pairs produce the same population sex ratio if they fulfil the condition

$$
b=a * 2 * c^{2}+c
$$

However, $a-b$ pairs that produce the same sex ratio $s$ don't necessarily produce the same evolutionary rate $u$. According to Equation (5) the $a-b$ pairs of $0.5-2.0$ and 1.0-3.0 both produce a sex ratio $s=1$. Using the same pairs in Equation (7) with an $\alpha$ of 2, produces $u=1.25$ for the first pair and $u=1.33$ for the second pair. The evolutionary rate of an E population can, therefore, not be directly deducted from the sex ratio alone without knowing the demographic details of the specific population.

In obligatory sexual populations, the sex ratio can only influence the evolutionary rate of a population via drift effects and the average evolutionary rate $u$ is largely the average between the male and the female mutation rate if inheritance operates in a Mendelian way $\left(u=\frac{1+\alpha}{2}\right)$. Without 
Mendelian inheritance, as in the case of $R$. esculenta, this is not the case. Figure $3 \mathrm{a}-\mathrm{d}$ show how the neutral evolutionary rate of an $\mathrm{E}$ population depends on the combination of $a, b$ and $\alpha$ and how much the evolutionary rate differs from the one of a similar sexual species. If male and female mutation rates are equal $(\alpha=1, \log 10(\alpha)=0)$, the sex ratio cannot have an influence on the evolutionary rate. In this case the evolutionary rate of an $\mathrm{E}$ population would not differ from the one of a comparable sexual species. If males have a higher mutation rate than females (Fig. 3a-d) the particularities of the $R$. esculenta offspring production can have quite dramatic effects on the evolutionary speed of $R$. esculenta. If males are only half as successful in siring offspring as females $(\log 2(a)=-1)$, the reduction of the evolutionary speed of $\mathrm{E}$ autosomes compared to a sexual species is particularly pronounced. This difference in $u$ increases with increasing $\alpha$ and decreasing male survival $b$. If $\mathrm{L}$ females produce about half as many eggs as $\mathrm{E}$ females $(\log 2(a)=-1)$ and $\mathrm{E}$ males and females survive equally well $(\log 2(b)=0)$, and with the high male to female mutation rate that is characteristic for species with external fertilization ( $\alpha=10$, Fig. 3d), then the evolutionary speed of $\mathrm{E}$ autosomes drops down to $57 \%$ of the value for $R$. lessonae autosomes, Even if $\mathrm{L}$ females produce as many eggs as $\mathrm{E}$ females and, hence, $\mathrm{E}$ males and females had the same reproductive success $(\log 2(a)=0)$, the evolutionary speed would be only $70 \%$ of the speed of a sexual species. While the effect of the relative male survival $(b)$ on the neutral evolutionary rate $(u)$ is relatively small, the relative male reproductive success $(a)$ has a pronounced influence: the lower $a$, the lower is $u$ of $\mathrm{E}$ autosomes compared to autosomes of a sexual species. For both, $b$ and $a$, effects on $u$ increase with increasing $\alpha$.

\section{Discussion}

Sex ratios

Our mathematical analysis shows how sex ratio and neutral evolutionary rates in R. esculenta (E) depend on the relative reproductive success $(a)$ and survival (b) of $\mathrm{E}$ males compared to $\mathrm{E}$ females. For sex ratio, the model predictions (Fig. 2) can be directly tested with empirical data from our own lab. Averaged over two studies (Holenweg Peter, 2001; Anholt et al., 2003), survival is higher for $\mathrm{E}$ males than for $\mathrm{E}$ females $(b=1.29)$. Conversely, reproductive success is higher in E females than in E males. Abt (2003) analyzed 84 clutches from mixed species matings; 35 originated from $L$ females mating with $E$ males and 49 from $\mathrm{E}$ females mating with $\mathrm{L}$ males (ratio $=0.71$ ). Average clutch sizes were 780 eggs for $\mathrm{L}$ and 1070 eggs for $\mathrm{E}$ females (ratio $=0.73$ ). The product of these 

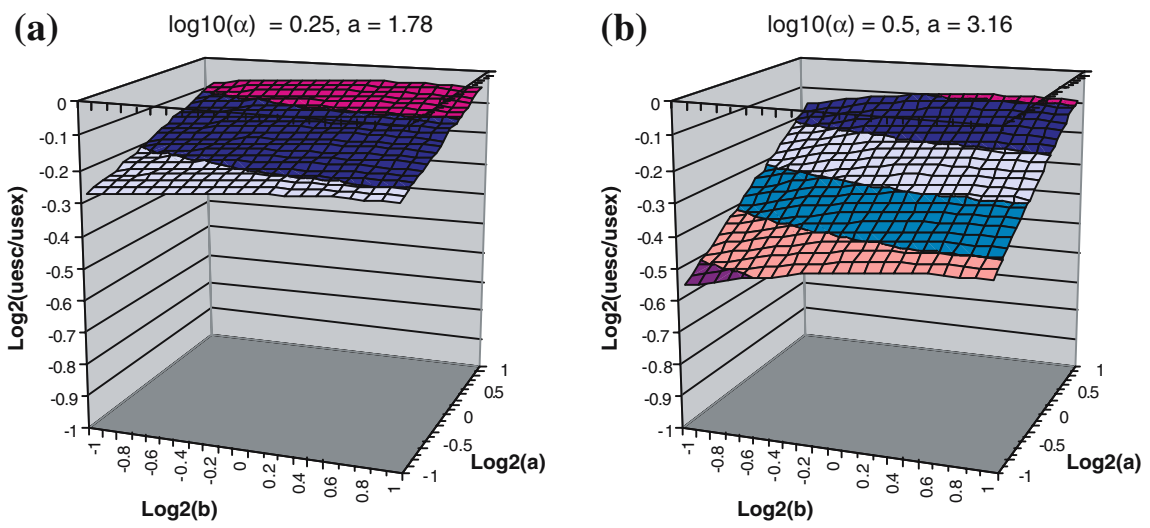

(c)

c) $\log 10(\alpha)=0.75, a=5.62$

(d)

d) $\log 10(\alpha)=1, \quad a=10$


Figure 3. Comparison of the evolutionary rate between $R$. esculenta (E) and a comparable sexual water frog species for four different values of the male to female mutation rate $(\alpha)$. The vertical axis shows the fraction of the E evolutionary rate compared to a sexual species with the same $\alpha$. If $\log 2(U$ esc $/ U$ sex $)=-1$, then the E population (precisely the transmitted R-genome part) evolves half as fast as the sexual species. Note that the horizontal axes for $a$ and $b$ are switched from Figure 2 for better readability of the results.

two ratios gives the reproductive success of $\mathrm{E}$ males relative to $\mathrm{E}$ females $(a=0.52)$. Entering these $a$ and $b$ values into Equation (5) yields an $s$ value of 0.73 . This is equivalent to a female proportion of $58 \%$, a value that is pretty close to the $62 \%$ observed by Holenweg and Abt (unpubl. data) in the same area, where reproductive success and survival were recorded.

Sex specific reproductive success and survival are likely to vary with environmental conditions. Sex differences in survival, for instance, can differ markedly between ponds (Holenweg Peter, 2001). The extent will, among other factors, depend on how much males are exposed to predators when defending or fighting over territories or calling at breeding ponds (Rand et al., 1997; Schwartz et al., 2000). Sex differences in reproductive success are affected by fecundity differences (i.e. clutch size) between $\mathrm{L}$ and $\mathrm{E}$ females and the mating 
and fertilization success of $\mathrm{E}$ males relative to $\mathrm{L}$ males (Helliegel and Reyer, 2000; Som et al., 2000; Reyer et al., 2004). Again, female fecundity and male fertilization rate are likely to vary with environmental conditions such as food resources and predation pressure, as well as with species composition and body size distribution in the mixed $\mathrm{L} / \mathrm{E}$ population. With respect to female fecundity, $\mathrm{L} / \mathrm{E}$ clutch size ratios in Polish populations were found to be around 0.30 (Berger and Uzzell, 1980; Juszczyk, 1974, cited from Günther, 1990), rather than around the 0.73 reported by Abt (2003) for her Swiss study population. To some extent, these differences in relative fecundity per reproductive event may be compensated by more or less regular breeding: in the same Swiss population $\mathrm{E}$ females skipped more often a reproductive season than $\mathrm{L}$ females (Reyer et al., 2004). Clutch sizes are further modified by 'cryptic' female choice, i.e. the ability of both female types to adjust clutch size in relation to the male type (L or E) they are amplexed by (Reyer et al., 1999). With respect to male mating success Blankenhorn (1977) observed an E/L mating ratio of 0. 55, instead of the 0.71 reported by Abt (2003), and Bergen et al. (1997) found that mating frequencies of $\mathrm{E}$ females were directly related to the proportion of $\mathrm{L}$ and $\mathrm{E}$ males in an experimental population. Moreover, mating frequencies alone may not be a reliable measure of male reproductive success, because $\mathrm{E}$ males show varying degrees of reduced fertility, ranging from equal fertilization rates of eggs by E- than by L-sperm to total sterility of E males (Günther, 1990; Reyer et al., 2003).

All these factors will affect $a$ and $b$ and, consequently, the male/female ratio $s$ (see Equation 5 and Fig. 2). Therefore, it is not surprising that a wide range of sex ratios have been reported for LE populations, e.g. 0.03 from the Danube River drainage system on the Balkan, 0.43 from Bavaria, 0.67 from the Baltic Sea in Poland and 1.06 from the Adriatic Sea in Italy (Berger et al., 1988; Zahn, 1997). Whether the observed $s$ values come close to the ones predicted by our model, can presently not be tested, because for none of these populations do we have sufficient data to estimate sex specific reproductive success $(a)$ and survival $(b)$.

\section{Evolutionary rates}

We have even less data to test the second prediction from our model, namely that the neutral evolutionary rate in the hemiclonal $R$. esculenta population (more precisely of the R-genome part) is lower than the corresponding rate in a comparable sexual population and that the extent of the deviation directly depends on a combination of male/female ratios in reproductive success $(a)$, survival $(b)$ and mutations rate $(\alpha)$ (Fig. 3a-d). Such a tight coupling between ecological/demographic characteristics and the evolutionary properties of a species is unusual and only possible because - contrary to normal sexual 
species - in $R$. esculenta the male reproductive success is independent of the female reproductive success and the males only produce daughters. Since in E populations, males and females recruit their offspring independently by mating with $R$. lessonae, the genetical state of the next generation depends on how much of this next generation originates from $\mathrm{E}$ males vs. how much from $\mathrm{E}$ females. If males and females differ in their mutagenic properties, everything that affects this ratio will also affect the micro-evolutionary peculiarities of an E population.

Although genetic data for testing the direct link between environment and evolutionary speed are lacking for $R$. esculenta, the results of our mathematical analysis (Fig. 3a-d) throw new light on the ongoing debate whether hemiclonal reproduction provides enough genetic diversity to guarantee ecological success and evolutionary longevity (Schmidt, 1993; Milinski, 1994). For asexually reproducing organisms, persistence in variable environments can be achieved in two different ways: either through a general-purpose genotype (Baker, 1965; Parker et al., 1977; Lynch, 1984) or by a high level of clonal diversity, which allows asexuals to occupy different niches (Vrijenhoek, 1979, 1984; Case and Taper, 1986). Since a general purpose genotype, too, can only arise through selection from a number of initial genotypes, both models require that clones either evolve fast enough or are frequently created de novo.

Both options are available to $R$. esculenta. With a sexual $\mathrm{L}$ and a clonally transmitted $\mathrm{R}$ genome the hybrid is hemiclonal and, hence, has an evolutionary speed lying between the speed of an asexual population and that of a sexual population. Thus, clonal diversity through new mutations is more easily achievable than in purely asexual species. On the other hand, the reduced average mutation rate compared to a comparable sexual species if $\alpha$ is high (Fig. 3c, d), lowers the occurrence and accumulation of deleterious mutations through Muller's ratchet (Som, in preparation). Thus the evolutionary potential of $R$. esculenta and the few other hybridogenetic species may not be as gloomy as suggested by Milinski (1994).

The results of our mathematical analysis emphasize the importance of field data in the study of evolution, since the micro-evolutionary properties of $R$. esculenta populations can only be assessed with the knowledge of the sex biases in survival ( $b$ ) and reproductive success $(a)$. Since the effects of $a$ and $b$ depend on $\alpha$, we also must know the male/female mutation rates which are likely to be species-specific and higher in species with external than in those with internal fertilization. They may also vary with the extent of sexual selection (Moeller and Cuervo, 2003).

Moreover, our results have implications for practical work on $R$. esculenta and similar hemiclonal systems. Microsatellites, the manifestation of neutral mutations par excellence, evolve fast enough, that the effect of the here described mutation rates plays a role, even if hemiclones as 'species' should be short-lived. The thought, that microsatellites on the clonal part of a genome 
(the R-part in R. esculenta) might evolve only half as fast, or even slower, than the very same microsatellites in a sexual genome (the $\mathrm{R}$ in $R$. ridibunda), and therefore have only half the resolution, is quite intriguing.

\section{Acknowledgements}

We thank C. Vorburger, T. Garner, R. Altwegg, B. Anholt and J. Van Buskirk for discussions and advice, and two anonymous reviewers for helpful comments. The whole mob of the Ecology department at the University of Zurich created a wonderful and unique work atmosphere. Our work was financially supported by Swiss National Science Foundation grant 31-40688.94 to H.-U. R.

\section{References}

Abt, G. (2003) Pond use, patterns of reproduction and juvenile recruitment in a mixed waterfrog population. $\mathrm{PhD}$ thesis, University of Zurich, Switzerland.

Abt, G. and Reyer, H.-U. (1993) Mate choice and fitness in a hybrid frog: Rana esculenta females prefer Rana lessonae males over their own. Behav. Ecol. Sociobiol. 32, 221-228.

Anholt, B.R., Hotz, H., Guex, G.-D. and Semlitsch, R.D. (2003) Overwinter survival of Rana lessonae and its hemiclonal associate. R. esculenta. Ecology 84, 391-397.

Baker, H.G. (1965) Characteristics and modes of origin of weeds. In H.G. Baker and G.L. Stebbins (eds) Genetics of Colonizing Species. Academic Press, New York, pp. 147-172.

Bergen, K., Semlitsch, R.D. and Reyer, H.-U. (1997) Hybrid female matings are directly related to the availability of Rana lessonae and Rana esculenta males in experimental populations. Copeia 1997, 275-283.

Berger, L. (1976) Hybrids of B-2 generations of European water frogs (Rana esculenta complex). Ann. Zool. 33, 201-214.

Berger, L. (1977) Systematics and hybridization in the Rana esculenta complex. In D.H. Taylor and S.I. Guttman (eds) The Reproductive Biology of Amphibians. Plenum Press, New York, pp. 376-388.

Berger, L. and Uzzell, T. (1980) The eggs of European water frogs of the Rana esculenta-complex and their hybrids. Folio Biol. 28, 3-26.

Berger, L., Uzzell, T. and Hotz, H. (1988) Sex determination and sex ratios in western Palearctic water frogs: XX and XY hybrids in the Pannonian Basin? Proc. Acad. Nat. Sci. Phila. 140, 220-239.

Blankenhorn, H.J. (1977) Reproduction and mating behavior in Rana lessonae-Rana esculenta mixed populations. In D.H. Taylor and S.I. Guttman (eds) The Reproductive Biology of Amphibians. Plenum Press, New York, pp. 389-410.

Case, T.J. and Taper, M.L. (1986) On the coexistence and coevolution of asexual and sexual competitors. Evolution 40, 366-387.

Charlesworth, B. and Charlesworth, D. (1997) Rapid fixation of deleterious alleles can be caused by Muller's ratchet. Genet. Res 70, 63-73.

Ellegren, H. and Fridolfsson, A.K. (2003) Sex-specific mutation rates in salmonid fish. J. Mol. Evol. 56, 458-463.

Engeler, B. and Reyer, H.-U. (2001) Choosy females and indiscriminate males: mate choice in mixed populations of the water frogs Rana lessonae and Rana esculenta. Behav. Ecol. 12, 600-606.

Felsenstein, J. (1974) The evolutionary advantage of recombination. Genetics 78, 737-756. 
Graf, J.-D. and Müller, W.P. (1979) Experimental gynogenesis provides evidence of hybridogenetic reproduction in the Rana esculenta complex. Experientia 35, 1574-1576.

Günther, R. (1990) Die Wasserfrösche Europas. Neue Brehm-Bücherei, Wittenberg, Germany.

Guex, G.-D., Hotz, H. and Semlitsch, R.D. (2002) Deleterious alleles and differential viability in progeny of natural hemiclonal frogs. Evolution 56, 1036-1044.

Haldane, J.B.S. (1935) The rate of spontaneous mutation of a human gene. J. Genet. 31, 317-326.

Haldane, J.B.S. (1947) The mutation rate of the gene for haemophilia, and its segregation rates in males and females. Ann. Eugenic. 13, 262-271.

Hellriegel, B. and Reyer, H.-U. (2000) Factors influencing the composition of mixed populations of a hemiclonal hybrid and its sexual host. J. Evol. Biol. 13, 906-918.

Holenweg Peter, A.-K. (2001) Survival in adults of the water frog Rana lessonae and its hybridogenetic associate Rana esculenta. Can. J. Zool. 79, 652-661.

Hurst, L.D. and Ellegren, H. (1998) Sex biases in the mutation rate. Trends Genet. 14, 446-452.

Kirkpatrick, M. and Hall, D.W. (2004) Male-biased mutation, sex linkage, and the rate of adaptive evolution. Evolution 58, 437-440.

Lynch, M. (1984) Destabilizing hybridization, general-purpose genotypes and geographical parthenogenesis. Q. Rev. Biol. 59, 257-290.

Makova, K.D. and Li, W.H. (2002) Strong male driven evolution of DANN sequences in humans and apes. Nature 416, 624-626.

McVean, G. (2000) Evolutionary genetics: what is driving male mutation? Curr. Biol. 10, R834-R835.

Milinski, M. (1994) Hybridogenetic frogs on an evolutionary dead end road. Trends Ecol. Evol. 9, 62.

Miyata, T., Hayashida, H., Kuma, K., Mitsuyasu, K. and Yasunaga, T. (1987) Male-driven molecular evolution: a model and nucleotid sequence analysis. Cold Spring Harb. Sym. 52, 863-867.

Moeller, A.P. and Cuervo, J.J. (2003) Sexual selection, germline mutation rate and sperm competition. BMC Evol. Biol. 3.

Muller, H.J. (1964) The relation of recombination to mutational advance. Mut. Res. 1, 2-9.

Parker, E.D. Jr., Selander, R.K., Hudson, R.O. and Lester, L.J. (1977) Genetic diversity in colonizing parthenogenetic cockroaches. Evolution 31, 836-842.

Rand, A.S., Bridarolli Dries, M.E. L. and Ryan, M.J. (1997) Light levels influence female choice in Tungara frogs: Predation risk assessment? Copeia 1997, 447-450.

Redfield, R.J. (1994) Male mutation rates and the cost of sex for females. Nature 369, 145-147.

Reyer, H.-U., Frei, G. and Som, C. (1999) Cryptic female choice: frogs reduce clutch size when amplexed by undesired males. P. Roy. Soc. Lond. B 266, 2101-2107.

Reyer, H.-U., Niederer, B. and Hettyey, A. (2003) Variation in fertilisation abilities between hemiclonal hybrid and sexual parental males of sympatric water frogs (Rana lessonae, R. esculenta, R. ridibunda). Behav. Ecol. Sociobiol. 54, 274-284.

Reyer, H.-U., Wälti, M.-O., Bättig, I., Altwegg, R. and Hellriegel, B. (2004) Low proportions of reproducing hemiclonal females increase the stability of a sexual parasite-host system (Rana esculenta, R. lessonae). J. Anim. Ecol. 73, 1089-1101.

Roesli, M. and Reyer, H.-U. (2000) Male vocalization and female choice in the hybridogenetic Rana fessonae/Rana esculenta complex. Anim. Behav. 60, 745-755.

Schmidt, B.R. (1993) Are hybridogenetic frogs cyclical parthenogens? Trends Ecol. Evol. 8, 271-273.

Schultz, R.J. (1969) Hybridization, unisexuality, and polyploidy in the teleost Poeciliopsis (Poeciliidae) and other vertebrates. Am. Nat. 103, 605-619.

Schwartz, J.J., Bee, M.A. and Tanner, S.D. (2000) A behavioral and neurobiological study of the responses of gray treefrogs, Hyla versicolor, to the calls of a predator, Rana catesbeiana. Herpetologica 56, 27-37.

Semlitsch, R.D. and Reyer, H.-U. (1992) Performance of tadpoles from the hybridogenetic Rana esculenta complex: interactions with pond drying and interspecific competition. Evolution 46, 665-676. 
Som, C., Anholt, B.R. and Reyer, H.-U. (2000) The effect of assortative mating on the coexistence of a hybridogenetic waterfrog and its sexual host. Am. Nat. 156, 34-46.

Tunner, H.G. (1974) Die klonale Struktur einer Wasserfroschpopulation. Z. Zool. Syst. Evol. 12, 309-314.

Uzzell, T., Hotz, H. and Berger, L. (1980) Genome exclusion in gametogenesis by an interspecific Rana hybrid: evidence from electrophoresis of individual oocytes. J. Exp. Zool. 214, 251-259.

Vorburger, C. (2001) Fixation of deleterious mutations in clonal lineages: evidence from hybridogenetic frogs. Evolution 55, 2319-2332.

Vrijenhoek, R.C. (1979) Factors affecting clonal diversity and coexistence. Am. Zool. 19, 787-797.

Vrijenhoek, R.C. (1984) Ecological differentiation among clones: the frozen niche variation model. In K. Wöhrmann and V. Löschke (eds) Population Biology and Evolution. Springer-Verlag, Berlin, Germany, pp. 217-231.

Zahn, A. (1997) Untersuchungen zum Rana kl. esculenta - lessonae-Komplex in Oberbayern. Salamandra 33, 79-88. 